\title{
FUERZA, MUNDO SUPRASENSIBLE Y LEY: SOBRE EL CONCEPTO DE FENÓMENO (ERSCHEINUNG) EN EL CAPÍTULO III DE LA FENOMENOLOGÍA DEL ESPÍRITU DE HEGEL
}

\author{
FORCE, SUPERSENSIBLE WORLD AND LAW: ON THE CONCEPT \\ OF APPEARANCE (ERSCHEINUNG) IN CHAPTER III \\ OF HEgEL'S PHENOMENOLOGY OF MIND
}

\author{
Agustín Palomar Torralbo \\ Universidad de Granada \\ agupalomar@yahoo.es
}

\begin{abstract}
Resumen: Este artículo tiene como objetivo aclarar desde una perspectiva fenomenológica el concepto de fenómeno en el capítulo de la Fenomenología del espíritu dedicado al entendimiento. En primer lugar, el texto muestra de qué modo el fenómeno se define como la totalidad de lo que aparece en el contexto del concepto de fuerza de la filosofía de la naturaleza. El artículo explicita a continuación cómo, a través de este concepto de fenómeno, el mundo suprasensible devine en objeto para el entendimiento y nombrado, por ello, como el verdadero fenómeno. A partir de aquí, en tercer lugar, el texto muestra, a su vez, cómo a través del mundo suprasensible, el mundo de las leyes de la naturaleza se constituye en objeto para el entendimiento y cómo estas leyes no pueden dar cuenta efectivamente del surgimiento de lo que aparece, mostrando, por tanto, ya desde aquí, la necesidad de un concepto filosófico de fenómeno.
\end{abstract}

Palabras clave: fenómeno, fuerza, mundo suprasensible, entendimiento

\begin{abstract}
The purpose of this article is to clarify from a phenomenological perspective the concept of appearance in the chapter of the Phenomenology of Mind dedicated to the concept of understanding. Firstly, the text shows how the concept of appearance is defined as the totality of seeming in the context of the concept of force within the philosophy of nature. Secondly, the article explains how the supersensible world, through this concept of appearance, turns into an object for understanding and now it is designated as the authentic appearance. Finally, the text shows how through the supersensible world, the world of the laws of nature is turned into an object for understanding and how these laws cannot effectively account for the emergence of what appears, thus, exhibiting since here the need for a philosophical concept of appearance.
\end{abstract}

Keywords: appearance, force, supersensible world, understanding 
No puede leerse un capítulo de la Fenomenología del espíritu. Leer un capítulo es leer la Fenomenología entera. Se dice que cuando uno ha terminado y ha cerrado el libro, si antes no capitulado en la empresa, está en el mejor momento para empezar de nuevo, y se dice que en el comienzo ya está de alguna manera su final. Pero, el problema de leer la Fenomenología, avanzando esta obra algunos de los temas que luego sistematizará la hermenéutica, es que quien la lee tiene que entrar en el juego de las perspectivas en las que va desarrollándose el saber. Leer fenomenológicamente la Fenomenología es tener la experiencia de ir superando cada una de las experiencias que tiene la conciencia en su camino hacia el saber absoluto. $Y$ es, en este sentido, en el cabe decir que no puede leerse la Fenomenología como si este camino estuviera trazado capítulo a capítulo. El mismo Hegel no lo hizo, y no quiso facilitar la tarea al lector, porque, en su lectura, la conciencia tiene más una experiencia del tiempo de la narración que de las partes en las que la obra se divide. En la Fenomenología, más que de capítulos que empiezan y terminan, nos las vemos con el tiempo y sus momentos, y cada momento ha de tomarse - esta es la exigencia específica- como un tiempo completo. Pero, aquí "completo" más que hacer referencia a todo el tiempo necesario para leerla, significa que cada uno de esos momentos no se sostiene ni puede sostenerse en sí mismo pero tampoco puede tomarse como tal si de alguna manera no se mantienen presentes los momentos precedentes $y$, desde esos momentos que se recapitulan en el acto de la lectura, no se avanza en la perspectiva de los que vendrán. Por ello, si tenemos que dar cuenta de uno de estos momentos hemos de re-iterar como lectores al menos el hilo que anuda los momentos en el tiempo de la lectura. Pero Hegel, que exige al lector la experiencia del camino de la fenomenología, lo ayuda, sin embargo, en su trabajo con esos textos escritos para nosotros für uns- en los cuales se recapitula lo ya alcanzado en la experiencia de la conciencia y se vislumbra lo que va a ser experienciado.

Para la lectura de nuestro capítulo, Hegel nos da el hilo con el que está formado el tejido que hemos de seguir para desovillar el texto: lo universal incondicionado (Unbedingt-Allgemeinen) como objeto del entendimiento. Esta expresión, en el primero de sus términos, nos recuerda esas primeras palabras 
del Prólogo donde Hegel sostiene que la filosofía existe en el elemento de lo universal que lleva dentro de sí lo particular. ${ }^{1}$

La filosofía nos descubre cómo en lo particular estaba ya lo universal, pero este saber de la filosofía sólo llega verdaderamente, como bien sabemos, al final. Es penoso el camino de la conciencia por llegar a este final y, sin embargo, es este esfuerzo, que tiene en su inicio sólo la inquietud por saber, el que dará el contenido de experiencia a la conciencia. La conciencia adquiere el saber de la experiencia en la medida en que encuentra la verdad de lo universal en lo particular que va apareciendo ante ella. De este modo, la marcha hacia el saber, que se describe en la Fenomenología, traza el camino de la experiencia de la conciencia, como superación de la experiencia inmediata en su particularidad, en la constatación de que en esta experiencia ya estaba presente el elemento universal que, en su mediación, la atravesaba. Esta superación, que va definiendo las diferentes figuras del saber, se hace lógicamente posible porque la conciencia, como conciencia intencional, va tomando como objeto de experiencia las relaciones que median entre las cosas hasta llegar a ver-se en ellas como la que las constituye, y, por tanto, como la que ya estaba presente mediando en aquellas relaciones que, por ello, ahora son vistas para la propia conciencia como inmediatas. Así puede decirse que la experiencia que la conciencia va haciendo en el camino de la fenomenología es la que corresponde a las relaciones constituyentes de lo que toma en cada momento como objeto suyo, un objeto que va tras-figurándose hasta la completa mediación de sí mismo que se da en el saber absoluto. En nuestro estudio veremos cómo, en el paso de la conciencia a la autoconciencia, tiene lugar una primera definición del fenómeno que podemos adjetivar de "fenomenológica". ${ }^{2}$

\footnotetext{
${ }^{1}$ Cf. G. W. F. Hegel, Fenomenología del espíritu, trad. W. Roces, , México D.F.: F.C.E., 1996, p. 7. [Se cita por esta traducción si no se dice otra cosa. También se tendrán en cuenta las otras dos ediciones y traducciones que existen en castellano: G. W. F. Hegel, Fenomenología del espíritu, trad. A. Gómez Ramos, Madrid: Abada Editores, 2010; G. W. F. Hegel, Fenomenología del espíritu, trad., M. Jiménez Redondo, Valencia: Pretextos, 2006. Para el texto en alemán se ha utilizado: G. W. F. Hegel, Phänomenologie des Geistes, Frankfurt am Main: Suhrkamp Taschenbuch Verlag, 1970.]

2 Un estudio de la fenomenología de Heidegger y de Hegel a partir de los conceptos de Phänomen y Erscheinung, puede encontrarse en: Á. Xolocotzi Yañez, "Aparecer y mostrarse. Notas en torno a la fenomenología' en Hegel y Heidegger", Eidos, 12, (2010), pp. 10-27.
} 


\section{LO UNIVERSAL INCONDICIONADO Y LA FUERZA}

Efectivamente, como hemos apuntado, el concepto de fenómeno se pone en juego para la conciencia en la correlación entre lo universal incondicionado y el entendimiento. Pero, antes de entrar en la experiencia de la conciencia con este objeto, recordemos que la universalidad ya estaba presente en la conciencia de la certeza sensible como la universalidad del lenguaje: "Como un universal -dice allí Hegel- enunciamos también lo sensible". ${ }^{3} \mathrm{Y}$ recordemos que también estaba presente en la figura de la percepción en tanto que el objeto de la conciencia que es la cosa, considerado en su unidad, en lo que es para sí, se nos mostraba como lo contrario de sí mismo, esto es, como un objeto que es "para sí en tanto que es para otro y para otro en tanto que es para sí". ${ }^{4}$ Pero, es de este modo como la cosa en su relación con otras cosas deviene un algo universal, es decir, un objeto que se constituye como tal en una relación universal que trasciende las determinaciones de cada cosa singularmente considerada. La cosa, objeto de la percepción, se presenta así para la conciencia en una determinación universal bajo la que antes no era vista, y con ello, se nos figura en una unidad que no podía ser captada en la percepción particular. Por esta razón puede Hegel afirmar que la universalidad nace para nosotros como principio general de la percepción. ${ }^{5}$

Ahora bien, esta universalidad de la conciencia en la percepción ha de dejar paso a la universalidad de la relación de las cosas en su respectividad plural, donde la relación es vista través de la unidad que liga en sí la pluralidad de un modo menos general, esto es, de un modo menos abstracto que la pura relación de contraposición en la que están las cosas en el mundo sensible. La cuestión, como ya dijo Hegel en el capítulo II, es que la universalidad de la percepción está afectada por la sensibilidad, condicionada por ella, y, por tanto, no era todavía verdadera universalidad. ${ }^{6}$ Esta sólo aparece cuando la conciencia tiene como objeto suyo uno cuya universalidad ya no venga afectada por la relación de contra-posición de los objetos sensibles. Tal cosa tiene su lugar en la figura del entendimiento que, a diferencia de la de la figura de la percepción, tiene la

\footnotetext{
${ }^{3}$ Fenomenología del espíritu, cit., p. 65.

${ }^{4}$ Ibíd., p. 79.

${ }^{5}$ Cf. Ibíd., p. 71.

${ }^{6}$ Cf. Ibíd., p. 80.

Investigaciones Fenomenológicas, n. 13, 2016.
} 
capacidad de ver, tras esa relación de contraposición, una unidad subyacente, una unidad que se presenta ante la conciencia como una universalidad incondicionada.

Pues bien, este re-cuerdo o re-iteración de la experiencia de la conciencia en torno a la constitución de la cosa, que se expone en los dos primeros capítulos, se hace presente en los cuatro primeros párrafos del capítulo III: el ser para sí y el relacionarse hacia otro en general $-z u$ Anderem sich zu verhalten überhaupt- constituye la esencia del contenido que tiene como verdad la de ser un universal incondicionado. Ahora bien, este resultado que se producía como consecuencia de la contraposición absoluta del ser para sí y del ser para otro, parece que es meramente formal: una cosa es para sí misma en tanto que es para otra, y esta es con respecto a otra anterior y esta, a su vez, respecto a otra, y así sucesiva e infinitamente. Pero, esta no es sólo una relación formal, sino también de contenido que da su objeto a la conciencia o, mejor dicho, es una relación en la que, a través de la mera relación de contraposición del ser para sí en tanto que ser para otro, se pone en juego el contenido mismo de la conciencia como el modo en el que se nos muestran los entes. Se trata de un contenido universal porque "no puede haber allí ningún otro contenido que por su especial constitución se sustraiga al retorno a esta universalidad incondicionada". ${ }^{7}$ Ningún objeto puede ser tomado para la conciencia como más universal que la relacionabilidad misma en la que los entes están referidos y vinculados entre sí en su oposición. Pero, ¿a qué aludimos aquí? ¿Cuál es el contenido que aparece mediando esa aparente relación formal de la conciencia? ¿Hay alguna esencia que nos haga comprender ontológicamente cómo se constituyen la relación de las cosas en su respectividad?

Tal esencia viene dada por el movimiento. Efectivamente, en el movimiento el ser para sí de otro, es decir, la esencia está en ser uno paso del otro, en ser uno tránsito del otro. Es la fuerza la que nos hace ver la relacionabilidad en su dinamismo interno. En este sentido, Fink ha escrito que "con el concepto de fuerza Hegel puede referirse a la interna movilidad en la estructura del ente y, ante todo, puede hacer comprensible la multiplicidad de las determinaciones

\footnotetext{
${ }^{7}$ Ibíd., p. 83.
} 
categoriales a partir de un principio organizador". ${ }^{8}$ En la tradición metafísica, la cuestión del principio organizador de la multiplicidad en la que se nos presenta la realidad fue enunciada bajo el problema de las categorías. Según Fink, para Hegel la fuerza es la categoría fundamental en la constitución de la cosa, de tal modo que todas las demás categorías son sólo "formas configuradas a partir de este principio fundamental". ${ }^{9}$ Hegel ganaría con ella fundamentalmente tres cosas: superar la concepción estática de la cosa, conjugar los pensamientos contradictorios en torno a ella, $y$, sobre todo, abrir una "dimensión profunda en la constitución del ser de la cosa". ${ }^{10}$ Que el ser de la cosa, su entidad, sea fuerza y no substancia (hypokeimenon) implica que el ser mismo de la cosa es movimiento. Bajo esta interpretación ontológica, Fink llega a decir lo siguiente: "El ser del ente acontece, ocurre. Es el acontecer de un movimiento, un ejercer la fuerza, un juego-de-fuerzas". ${ }^{11}$ Ya antes Heidegger, partiendo de las categorías denominadas "dinámicas" de la Crítica de la razón pura, había sostenido en su lectura de la Fenomenología del espíritu, en un sentido próximo al de Fink, que la coseidad de lo que se muestra a la conciencia en la percepción aparece como objeto del entendimiento en la relación de fuerza de la acción recíproca. "El rótulo de la relación - afirma Heidegger- es la expresión 'fuerza' ". ${ }^{12}$ Pero esta interpretación, que pone en el centro de la comprensión del ser a la fuerza, contradice nuestra manera natural de considerar el ser del ente. De forma natural, el movimiento es considerado en referencia a algo quieto o en reposo, lo cual siempre ha sido visto como la verdadera esencia del ente. Sin embargo, aquí el ser es esencialmente relación que se da en un movimiento que no tiene una referencia o quietud en su constitución interna. Pero, ¿de dónde surge esta comprensión del ser en su interna motilidad?

Aunque se ha señalado que no es fácil localizar las referencias históricas que están presentes en este capítulo, ${ }^{13}$ está claro que Hegel la hace surgir del nuevo paradigma científico "marcado por el desarrollo de la biología, la electri-

\footnotetext{
${ }^{8}$ E. Fink, Hegel. Interpretaciones fenomenológicas de la Fenomenología del Espíritu, Barcelona: Herder, 2011 , p. 156.

${ }^{9}$ Ibíd.

${ }^{10}$ Ibíd., p. 167.

${ }^{11}$ Ibíd.

12 M. Heidegger, La fenomenología del espíritu de Hegel, Madrid: Alianza Universalidad, 1992, p. 188.

${ }^{13}$ Cf. J. Stewart, La unidad de la Fenomenología del espíritu de Hegel, México D. F.: Universidad Iberoamericana, 2014, p. 127.
} 
cidad y la química", ${ }^{14}$ pero también del pensamiento en torno a la naturaleza de filósofos como Locke, Leibniz, Kant y Schelling. ${ }^{15}$ La conciencia entra en el capítulo del entendimiento con la experiencia que ella tiene en la modernidad de los fenómenos de la naturaleza. Hegel toma este saber en torno a la naturaleza para hacer de él objeto de experiencia de la conciencia como un fenómeno que se le muestra a la conciencia según las leyes de la ciencia física moderna que tiene en la fuerza su concepto central. La fuerza permite a Hegel pensar los fenómenos naturales como lo universal dado al entendimiento que ya no está sometido a la condición sensible de la cosa particular. Pero, de todas las referencias históricas señaladas, esta tesis de Hegel tiene su origen más cercano en el modo en el que Leibniz, para salir al paso de las aporías de la mecánica cartesiana, concibe la substancia como fuerza. ${ }^{16}$ Efectivamente, se ha señalado que la dinámica de Leibniz es más próxima a Hegel que la mecánica de Newton y está "profundamente vinculada a una proyección ontológica que Hegel integra sin mayores dificultades en su propia ontología". ${ }^{17}$ Por tanto, lo que Hegel expondría en este capítulo de la Fenomenología sería una concepción de la substancia caracterizada como fuerza y potencia, como actividad y proceso, haciendo suya la noción axial de la dinámica de Leibniz que es la de fuerza. ${ }^{18}$

Pero, a pesar de esta proximidad, habría una notable diferencia: Hegel llevaría a cabo una radicalización de la propia concepción de la dinámica en Leibniz. Como afirma A. Pérez Quintana, en la dinámica no es necesario recurrir a una fundamentación extrínseca de la fuerza misma: "Hegel señala una y otra vez que con la armonía preestablecida el protagonismo en la actividad pasa a ser prerrogativa de un principio transcendente" ${ }^{19}$ La crítica, por tanto, de Hegel

\footnotetext{
14 J. M. Ripalda, "Fuerza y entendimiento. El conflicto de la especulación y la realidad", en F. Duque, Hegel. La odisea del espíritu, Madrid: Círculo Bellas Artes, 2010, p. 77.

${ }^{15} \mathrm{~L}$. Siep, El camino de la Fenomenología del espíritu. Un comentario introductorio al Escrito sobre la Diferencia y la Fenomenología del Espíritu de Hegel, Madrid: Anthropos, 2014, p. 92. Ripalda ha señalado, por su parte, que el problema de la fuerza dentro de la filosofía idealista de la naturaleza tiene su origen en el Apéndice de la Crítica de la razón pura donde Kant utiliza la noción de fuerza para explicar la causalidad (cf. J. M. Ripalda, op. cit., p. 77).

${ }^{16}$ Cf. G. W. Leibniz,"Nuevo sistema de la naturaleza y de la comunicación de la substancias así como de la unión que hay entre alma y cuerpo" en Id., Antología, Barcelona: Círculo de Lectores, 1997, p. 255.

${ }_{17}$ A. Pérez Quintana, "Teoría leibniziana de la fuerza y vicisitudes de la metafísica en la modernidad según la Fenomenología del espíritu de Hegel", en M. Sánchez Rodríguez y S. Rodero Cilleros (coord.), Leibniz en la filosofía y en la ciencia modernas, Granada: Comares, 2010, p. 560-561.

${ }_{18}$ Cf. A. Cardoso, "La fundación leibniziana de la dinámica", en N. Marín y S. Toledo (ed.), Leibniz y las ciencias empíricas, Granada: Editorial Comares, 2011, p. 266. Para la crítica de Leibniz al mecanicismo desde el concepto de fuerza viva -energía cinética-, puede verse: E. Álvarez Martino, El laberinto de la continuidad en G. W. Leibniz. El filósofo de los principios, Madrid: Biblioteca Nueva, 2011, pp. 39-80.

${ }^{19}$ Cf. Á. Pérez Quintana, op. cit., p. 563.
} 
a Leibniz se basa, en este punto, en que no deriva la relación entre las fuerzas de su recíproca y mutua oposición. Para ello, Leibniz tendría que haber tenido una concepción dialéctica de las fuerzas, según la cual, el dinamismo de la substancia se genera en la relación por oposición de unas substancias con otras. En su concepción de la fuerza, Hegel pondrá en el interior de la relación entre las substancias el fundamento que Leibniz pone fuera como armonía preestablecida. Pero, en segundo lugar, también el leibnizismo representa, a juicio de Hegel, un límite para el desarrollo del saber filosófico. Efectivamente, en la filosofía de Leibniz el espíritu se detendría en la figura de la conciencia del entendimiento sin poder transitar para llegar a saber-se como autoconciencia y como una autoconciencia que se despliega como tal en el enfrentamiento con lo otro extraño y negativo de ella misma que no puede verse representado en la conciencia, aunque, lo representado en ella, tenga su origen en el interior de la propia substancia concebida dinámicamente como fuerza viva. ${ }^{20}$

Pues bien, en la Fenomenología se encuentra el concepto de fuerza en el siguiente texto:

De donde se desprende que el concepto de fuerza deviene real al desdoblarse en dos fuerzas y también cómo llega a esto. Estas dos fuerzas existen como esencia que son para sí; pero su existencia es ese movimiento de la una con respecto a la otra en cuanto su ser es más bien un puro ser puesto por otro, es decir, en cuanto su ser tiene más bien la pura significación del desaparecer. ${ }^{21}$

El concepto de fuerza es realidad efectiva - wirklich - cuando la fuerza se desdobla en dos fuerzas, en un juego de ambas fuerzas (Spiel der beiden Kräfte). Aunque cada fuerza esté contenida para sí, su existencia es en la medida en que está una con respecto a la otra, o mejor dicho, sólo existe como fuerza en tanto que una está en movimiento con respecto a la otra. De este modo, la relación absoluta de alteridad, presente en la percepción, aparece ahora en la fuerza que se desdobla en un movimiento de fuerzas que se requieren respectivamente para ser tanto una como ambas. Una fuerza existe sólo como referencia a otra en el movimiento de una con otra. La fuerza es movimiento y el mo-

\footnotetext{
${ }^{20}$ La noción de fuerza viva en Leibniz hace posible comprender el tránsito de la física estática a la dinámica y la interrelación entre las categorías físicas y las metafísicas. Cf. F. León Florido, "Orígenes y génesis del concepto de 'fuerza viva' en la dinámica leibniziana", en Q. Racionero y C. Roldán (comp.), G. W. Leibniz. Analogía y expresión, Madrid: Editorial Complutense, 1994, pp. 325-326.

${ }^{21}$ Fenomenología del espíritu, cit., p. 87.
} 
vimiento, en la naturaleza, es fuerza. Pero, ¿cuál es el ser de la fuerza si esta es movimiento, si esta es, en la naturaleza, dýnamis? Pues bien, el ser de una fuerza es puesto por el ser de otra de tal modo que puede afirmarse que el ser de la fuerza reside o, más bien, es en la relación misma. Fenomenológicamente, esto significa que el ser de una fuerza que nos aparece es al mismo tiempo su desaparecer en otra o, mejor dicho, que en su aparecer una lleva ya dentro de sí el que sea un desaparecer en otra. Este "dentro de sí" alude a que en su esencia lleva ya la fuerza el ser fuerza a través de otra fuerza, que, a su vez, lleva en otra su aparecer o bien su desparecer. No hay, podríamos decir, nada más interno o esencial al objeto de la fuerza que el tener su modo de existencia propio en el modo de la existencia de otra. ${ }^{22}$ Pero, con ello, su aparecer es en virtud de otro aparecer y, por tanto, el primer aparecer es con respecto al segundo un desaparecer. Y lo mismo cabría decir del segundo y así sucesivamente. Por tanto, termina Hegel diciendo "no tienen de hecho ninguna substancia que las sostengan y mantengan". ${ }^{23}$ Bajo la fuerza, como el continuo y permanente movimiento, la experiencia que la conciencia tiene de la naturaleza es la de un constante y permanente aparecer y desaparecer. $Y$ esta es, hay que apuntarlo, la concepción primera del fenómeno como manifestación que responde a una concepción física de la naturaleza en la que los fenómenos naturales mismos se nos muestran reducidos a la fuerza como una aparecer y un desaparecer. Aquí, aunque la conciencia se ha superado como percepción recuérdese que en la percepción la conciencia mantiene la tesis de que el movimiento de las cosas tiene una permanencia y un asiento en las substancias-, tiene la experiencia natural de que los entes aparecen y desaparecen por su intrínseca relacionabilidad. Sin embargo, en su deseo por saber, el espíritu qui-

\footnotetext{
22 No hay en el pensamiento de Hegel una síntesis resultante de la oposición de las fuerzas, lo que sí encontramos es que las fuerzas, el juego de fuerzas, conforma una ontología y que, por tanto, alcanza a todo ente, abarcando la totalidad de lo que se da como "cosa" a la conciencia. En este sentido, la confrontación del pensamiento de Hegel con el de Deleuze no puede restringirse a señalar, como ha hecho Lucas Diel, que las fuerzas en Deleuze, siguiendo este a Nietzsche, están en un continuo combate mientras en Hegel esta oposición da como resultado una síntesis y una reconciliación. Cf. L. Diel, "La teoría de las fuerzas de Gilles Deleuze como alternativa al pensamiento dialéctico de Hegel", A Parte Rei, 70 (2010), pp. 1 y ss. Si hay una "superación" de las fuerzas esta estaría más bien en el poder de una ley universal que las hace aparecer en su oposición. La prueba de que no hay un tránsito de cuño dialéctico entre las cuestiones tratadas en este capítulo y las tratadas en el siguiente, dedicado a la autoconciencia, ha sido precisamente uno de los mayores escollos en la comprensión de este capítulo de la Fenomenología, cf. J. Stewart, op. cit., p. 127. Más que de una superación dialéctica, la concepción dinámica del ser lo que muestra finalmente es la inversión de la intencionalidad de la conciencia que pasa de apuntar a lo otro de sí a apuntar a ella misma, a descubrir-se como autoconciencia. Cf. L. Siep, op. cit., p. 94.

${ }^{23}$ Fenomenología del espíritu, cit., p. 88.
} 
siera hallar un principio o una ley subyacente que explicara o diera razón de ese aparecer y/o desaparecer. El espíritu, podríamos decir, requiere, en esta experiencia fenomenológica de la naturaleza, de Logos. Con una expresión de C. Moreno Márquez, podemos decir que la naturaleza se nos da primeramente como "nuestro espacio-de-Logos", el cual se nos ofrece "como apertura a un campo-de-presencia donde se hace luz para un pensamiento, un lenguaje o la praxis". ${ }^{24}$

Pero, previamente a la búsqueda de una legalidad para la naturaleza, la conciencia, ante ese abismo de una realidad convertida en puro fenómeno, en puro aparecer y desaparecer, se pregunta si no hay en el fondo del propio fenómeno una verdad, algo que no sea llevado por el movimiento de las fuerzas. ¿Qué verdad puede haber en tal concepción del ser de las cosas? Si toda la realidad es puro movimiento, si la conversión de todo el ámbito del ser objetivo se ha reducido, por mor de la fuerza, a puro fenómeno, ¿qué puede mantenerse en medio de ese movimiento? La respuesta de Hegel, en una expresión que Fink tilda de "giro sorprendente" 25 es: "La verdad de la fuerza se mantiene, pues, solamente como el pensamiento de ella". ${ }^{26}$ No se trata creo aquí principalmente, como dice el propio Fink, de que la fuerza real se desvele como pensamiento, ni que la fuerza sea sólo pensamiento en tanto que ella misma no puede ser captada de manera sensible, ni siquiera de que el entendimiento "penetre la apariencia superficial en la que el ente aparece al principio" ${ }^{27}$ concibiendo entonces que a la fuerza ha de subyacerle oculta una interioridad entre las cosas. Se trata más bien, de que la experiencia del carácter fenoménico del mundo lleva a la conciencia a plantearse la verdad de eso que aparece y desaparece. Pues bien, lo único que se mantiene en el movimiento de una realidad pensada a través de la fuerza es el propio pensamiento (Gedanke) de la fuerza, es decir, su contenido noématico. Del noema de árbol dice Husserl, para hacer ver su contenido ideal y no sensible, que no puede quemarse. ${ }^{28}$ Del noema de fuerza, análogamente, podría decirse que no puede moverse. La verdad de la

\footnotetext{
${ }^{24}$ C. Moreno Márquez, "El aparecer clausurado", en M. del Carmen Paredes Martín (ed.), Intencionalidad, mundo y sentido. Problemas de fenomenología y metafísica, Salamanca: Ediciones Universidad de Salamanca, 2003, p. 144.

${ }^{25}$ E. Fink, op. cit., p. 173.

${ }^{26}$ Fenomenología del espíritu, cit., p. 88.

${ }^{27}$ E. Fink, op. cit., p. 174.

${ }^{28}$ Cf. J. San Martín, Fenomenología y cultura en Ortega, Madrid: Tecnos, 1998, p. 128.
} 
realidad efectiva está en el propio pensamiento que la concibe. No puede pensarse la fuerza en su diferencia a las fases de su movimiento si no es en el concepto. Por tanto, la fuerza tiene su realidad sólo en el concepto. "Concepto y realidad de la fuerza - ha escrito Siep- se muestran tan indiferenciables como el dentro de sí o la disposición y exteriorización de la fuerza". ${ }^{29}$ El pensamiento de la fuerza ya no es simplemente la unidad que se mantiene en el concepto del juego de la fuerza, sino la unidad que es "su concepto como concepto"30 (ihr Begriff als Begriff). Hegel habla, como vemos, de esta verdad como de una unidad (Einheit). Esta unidad, en la que es pensada la categoría de fuerza, se constituye ahora en el verdadero objeto del entendimiento (Gegenstand des Verstandes), por cuanto supone que este ver es un ver que, penetrando a través de las fuerzas, contempla el interior de las cosas como lo que está en las diferentes fuerzas y, sin embargo, más allá de esta diferencia, en el concepto mismo. El fondo verdadero de las cosas, paradójicamente, es su concepto. Sólo en el concepto el aparecer de las fuerzas puede ser clausurado. Podría decirse que en la experiencia de la fuerza hace la conciencia una anticipación de lo que será para el espíritu el saber de lo absoluto: el contenido de lo que es sin más, no de lo que es de una manera u otra, de una perspectiva u otra, sino lo que simpliciter es, se da en el concepto. Sólo el ser puede ser lo que es en tu todo su aparecer en el concepto, puesto que solamente en él se rompe el vaivén de la experiencia como un aparecer que es un desaparecer y viceversa.

\section{EL FENÓMENO Y EL MUNDO SUPRASENSIBLE}

Por tanto, si el entendimiento tenía antes su objeto en la fuerza de forma inmediata, ahora, a través de ella, va a mirar, a leer dentro, de las cosas. Así, tras la exposición del concepto de fuerza, puede decir Hegel lo siguiente: "El término medio que enlaza los dos extremos, el entendimiento y lo interior, es el ser desarrollado de la fuerza que de ahora en adelante es para el entendimiento un desaparecer". ${ }^{31}$ Ahora bien, la pregunta es: ¿pero qué es entonces lo que aparece en cada aparecer? ¿Puede un aparecer ser aparecer si no desaparece? ¿Cómo hemos de entender el concepto de fenómeno desde el interior de la

\footnotetext{
${ }^{29}$ L. Siep, op. cit., p. 93.

${ }^{30}$ Fenomenología del espíritu, cit., p. 88.

31 Ibíd., p. 89
} 
fuerza que es su concepto? Pues bien, en primer lugar, lo que hay que afirmar es que el fenómeno, como aparecer, no es una apariencia (Schein). La apariencia, la mera apariencia, es de inmediato un no-ser (Nitchtsein). Pues bien, Hegel llama fenómeno (Erscheinung) a "la totalidad de lo que aparece" (Ganzes des Scheins). ${ }^{32}$ En esta especial caracterización de Hegel, el fenómeno, por tanto, no está vinculado a lo que aparece para luego desaparecer en un momento particular sino a una totalidad. El verdadero aparecer nos permite ver no un aparecer y luego un desaparecer, sino el aparecer como desaparecer y viceversa. Se trata, por tanto, de un aparecer que nos muestra la constitución íntima o interna por la que las cosas aparecen y desaparecen y, por tanto, del aparecer que está en todo aparecer pero también en todo desaparecer. Si no pudiera verse, en su extremo, el aparecer del desaparecer no se podría dar cuenta de la totalidad de lo que aparece. Fink, en su lectura fenomenológica de la Fenomenología, llega a identificar el fenómeno en Hegel como "el mostrarse mismo del ente" o como "el exponerse-a-la-conciencia". ${ }^{33}$ El fenómeno no es lo que aparece, sino aquello que hace posible la aparición de eso que aparece, conpareciendo, por ello, en todo aparecer y permaneciendo en su desparecer. En este sentido es la totalidad cuyo modo de ser sólo es visible al entendimiento.

Este concepto de fenómeno, que podríamos adjetivar de "fenomenológico", es ahora el verdadero objeto del entendimiento y constituye lo interior o la reflexión del juego de fuerzas para la conciencia. El juego de fuerzas se descubría ya en los momentos en los que se truecan respectivamente "sin descanso ni ser" inmediatamente en lo contrario de sí y, por ello, era "lo negativo desarroIlado". ${ }^{34}$ Sin embargo, ahora, en la fuerza como manifestación de la totalidad que aparece, se muestra algo positivo: "lo universal, el objeto que es en sí". ${ }^{35}$ Podríamos aludir a esta manifestación como al Entre del aparecer y desaparecer que hace posible ontológicamente, como hemos señalado, tanto lo uno como lo otro y que, fenomenológicamente, bien pudiera considerarse el mundo como objeto de experiencia en tanto que mundo natural para la conciencia. Ahora bien, en Hegel, este mundo-es-el-aparecer que sólo se da como tal para la con-

\footnotetext{
32 Ibíd.

${ }^{33}$ E. Fink, op. cit., p. 186-187.

${ }^{34}$ Fenomenología del espíritu, cit., p. 89.

${ }^{35}$ Ibíd. 
ciencia en la reducción de la experiencia de la naturaleza a su concepto ${ }^{36}$. O dicho de otra manera, en este concepto, para la propia conciencia, el mundo se da como un mundo suprasensible o un mundo verdadero. He aquí el pasaje decisivo de la Fenomenología:

En este verdadero interior, como lo universal absoluto depurado de la contraposición de lo universal y lo singular y devenido para el entendimiento, se revela ahora por vez primera, más allá del mundo sensible como el mundo que se manifiesta, un mundo suprasensible como el mundo verdadero, por encima del más acá llamado a desaparecer el más allá permanente; un en sí que es la manifestación primera de la razón, manifestación todavía, por tanto, imperfecta o solamente el puro elemento en que la verdad tiene su esencia. ${ }^{37}$

Efectivamente, Hegel habla en este texto, siguiendo a Platón, de mundo (Welt) como noēton eidos. ${ }^{38} \mathrm{El}$ mundo, considerado al modo platónico, es el concepto que recoge dentro de sí la estructura o el cómo de lo que se da a la conciencia como experiencia de la naturaleza. El mundo es la manifestación desde la que se da el aparecer y el desaparecer mostrando tanto lo uno como lo otro. Pero este es, justamente, el concepto fenomenológico de mundo que la tradición metafísica puso - por seguir con la metáfora espacial- "por encima del más acá"39 y que adjetivó como "suprasensible", porque, en definitiva, bien miradas las cosas, todo aparecer y todo desaparecer siempre está condicionado a lo sensible pero el mundo que hace posible lo uno y lo otro, ha de ser puro, es decir, no ha de estar afectado por el contenido de la sensibilidad. El mundo supra-sensible es el mundo opuesto al mundo sensible. Pero, al afirmar esto, sólo estamos dando una descripción negativa del mundo suprasensible en tanto que decimos de él lo que no es. Es, en este sentido, un "puro más allá para la conciencia". ${ }^{40}$ Como señala Hyppolite, Hegel toma aquí el noúmeno en sentido negativo. ${ }^{41}$

\footnotetext{
${ }^{36}$ Esta comprensión del texto de Hegel me ha sido sugerida en: C. Moreno Márquez, op. cit., pp. 148151.

${ }_{37}$ Fenomenología del espíritu, cit., p. 89-90.

${ }^{38}$ Cf. H. G., Gadamer, "Hegel y el mundo invertido" en Id., La dialéctica de Hegel, Madrid: Cátedra, 1988 , p. 56.

${ }^{39}$ Fenomenología del espíritu, cit., p. 89.

${ }^{40}$ Ibíd., p. 90.

${ }^{41}$ Cf. J. Hyppolite, Génesis y estructura de la fenomenología del espíritu de Hegel, Barcelona: Península, 1991 , p. 115
} 
Pero también aquí está la ocasión para el pensador suabo de llevar a cabo una crítica a este concepto de noúmeno que se sitúa para el entendimiento como el límite para los fenómenos, más allá del cual no es posible que la conciencia penetre para ver el modo como estos aparecen positivamente desde el noúmeno. El concepto negativo de noúmeno muestra aquí para el entendimiento un interior vacío, "pues es solamente la nada del fenómeno" ${ }^{42}$ y esta forma de concebir los fenómenos corresponde, refiriéndose Hegel evidentemente a Kant, "a quienes dicen que el interior de las cosas es incognoscible". ${ }^{43}$ Se trata, por tanto, de una manifestación de la razón que es incognoscible para el entendimiento. Si la razón es incapaz de conocer este interior y el entendimiento actúa para la razón como el límite del conocimiento, es porque, por principio, se ha situado el conocimiento en un terreno donde nada puede verse. No puede apresarse el contenido de lo universal incondicionado si el noúmeno sólo puede ser pensado negativamente como el resto de lo que no lleva en sí sensibilidad alguna. La determinación de este mundo como un más allá lleva ya en sí que el entendimiento se sitúe en él, en una maravillosa imagen de Hegel, como un "ciego en medio de la riqueza de ese mundo". ${ }^{44}$ Sin embargo, con esa finura que Hegel tiene para elaborar metáforas, en este bello texto, dice algo más: si el noúmeno es en sí pura manifestación inmediata o "pura luz", ${ }^{45}$ entonces tampoco podrá verse "la plétora de riquezas desplegadas ante él". ${ }^{46}$ Si el noúmeno es pura manifestación inmediata, que llega a nuestro entendimiento de un más allá inaccesible al conocimiento, entonces esa manifestación será a lo sumo una pura luz que ciega la mirada del que quiere ver el verdadero mundo.

Pero hay que reparar que, a reglón seguido, Hegel dice lo siguiente: "Si no se pudiera hacer nada con lo interior y el entrelazamiento con él mediante el fenómeno, no quedaría más camino que atenerse al fenómeno mismo...". ${ }^{47}$ Es decir, con lo interior, con ese fondo verdadero de las cosas que aparece abismarse en la nada, puede "hacerse" algo. No es, por tanto, un mundo inaccesible al entendimiento que sólo puede ser pensado mediante la vacía lógica de la no contradicción. El problema tal y como el kantismo lo dejó es que, establecida

\footnotetext{
${ }^{42}$ Fenomenología del espíritu, cit., p. 90.

${ }^{43}$ Ibíd.

${ }^{44}$ Ibíd.

45 Ibíd.

${ }^{46}$ Ibíd.

47 Ibíd.
} 
la diferencia entre fenómeno y noúmeno y situado el noúmeno como el interior verdadero que, sin embargo, está más allá del alcance del conocimiento del entendimiento, no quedaba más remedio que atenerse al fenómeno como tal "tomando como verdadero algo de lo que sabemos que no lo es". ${ }^{48} \mathrm{Si}$ esto fuera así, tendríamos que conformarnos con un saber que no es tal saber y resignarnos a tolerar esa rotura irreconciliable entre la verdad incognoscible y su manifestación como fenómeno. Pero, a su vez, si este fuera el caso, entonces esa vaciedad terminaría llenándose de sueños y ensueños engendrados por la propia conciencia fuera ya del rigor del concepto, quedando, de este modo, rebajada de la altura filosófica en la que se reconoce en su dignidad. Sin embargo, ahora, en la fuerza como manifestación de la totalidad que aparece, se muestra algo positivo. Su contenido pasaría a ser llenado, ante este horror vacui, con fenómenos que verdaderamente no lo son. Este es el tipo de apariencia engañosa que corresponde, en el terreno del entendimiento, a aquella otra con la que Hegel cerraba el capítulo II y que nacía de la "inseguridad de las cosas". ${ }^{49}$ Pero este engaño, ciertamente, nacido de la necesidad de dar un contenido al universal incondicionado, es mucho más grave que aquel otro, pues tiene su origen no ya en la falta de seguridad que la conciencia puede tener en las cosas, sino en una falsa apariencia de la que no puede determinarse su verdad. Bajo este texto, resuena explícitamente la crítica de Hegel a la separación kantiana entre la razón práctica y la razón teórica, y la necesidad de que el espacio para lo sagrado o para la fe tenga que venir dado de una sobreabundancia respecto a la de-limitación del saber en el conocimiento teórico.

Pero, dejemos a un lado la confrontación con Kant, que nos llevaría a estudiar este texto y este problema en el contexto de la dialéctica trascendental como una lógica de la ilusión, y pasemos a la cuestión que pende tras esta crítica a Kant: ¿De qué podría llenarse ese vacío? ¿Cómo es posible que lo interior, como fondo verdadero de las cosas, no esté vacío como una cáscara de nuez? ¿De qué está lleno lo suprasensible? O, como diríamos forzando de alguna manera la terminología husserliana, ¿en qué radicaría el cumplimiento de la intención de la conciencia en ese deseo de saber que ha quedado en nada? La respuesta de Hegel es la siguiente: lo suprasensible es aquello que se ha originado

\footnotetext{
${ }^{48}$ Ibíd.

49 Ibíd., p. 81.
} 
desde el fenómeno. Es el fenómeno lo que llena el mundo de lo suprasensible. Es el fenómeno lo que mediando entre el entendimiento y el interior de las cosas constituye lo suprasensible. Hay que subrayar que el fenómeno es la mediación que hace a lo suprasensible, es decir, lo suprasensible no es aquí un objeto inmediato a la conciencia, sino algo que se constituye en la mediación de su aparecer. Interpreta Hyppolite que aquí Hegel alude al "movimiento mediante el cual no cesa de desaparecer y de negarse a sí mismo -el mundo-". 50 "Lo que subsiste - dice a continuación- es la inestabilidad fenoménica, en el incesante cambio de sus momentos, es la diferencia, desde luego, pero la diferencia recibida en el pensamiento, convertida en universal". ${ }^{51} \mathrm{Y}$, a partir de aquí, lo suprasensible es nombrado por él como "la ley del fenómeno". ${ }^{52}$ Sin embargo, creo que aquí Hegel, más que referirse a lo suprasensible como aquello que subsiste dentro de la inestabilidad fenomenológica, alude a aquello que la hace posible y la constituye en su fondo: "la verdad de lo sensible y lo percibido es, empero, ser fenómeno", ${ }^{53}$ dice Hegel. La verdad de las dos figuras anteriores de la conciencia está en que es fenómeno, es aparecer, y esto, justamente, es lo suprasensible: que hay un lugar desde el que se puede determinar el modo como las cosas puedan aparecer para la conciencia de la certeza sensible y para la conciencia percipiente. El fenómeno requiere para su aparecer de la concurrencia del concepto. Así Hegel concluye lo siguiente: "Lo suprasensible es el fenómeno en cuanto fenómeno (Das Übersinniliche ist also die Erscheinung als Erscheinung)" ${ }^{54}$

\section{LA LEY Y LA DIFERENCIA UNIVERSAL}

Ahora bien, que se dé ya el fenómeno en cuanto fenómeno tiene para Hegel una repuesta determinada, clara y definitiva en el contexto del objeto del entendimiento: es la fuerza y la descripción fenomenológica de cómo esta fuerza se hace efectiva al tiempo que desaparece como juego de fuerzas particulares lo que explica cómo se llena o se constituye ese interior que para Kant permanecía vacío. Hegel, retomando lo que ya había dicho acerca de la relación inter-

\footnotetext{
50 J. Hyppolite, op. cit., p. 116.

${ }^{51}$ Ibíd.

${ }^{52}$ Ibíd.

${ }^{53}$ Fenomenología del espíritu, cit., p. 91.

${ }^{54}$ Ibíd. Edición alemana, p. 118. 
na de las fuerzas, sostiene que el fenómeno se muestra como fenómeno porque la realidad es ya fuerza y como fuerza se da para la conciencia, y la fuerza no es sólo lo que, por decirlo metafóricamente, impulsa al fenómeno a aparecer, a empujarlo para que salga a escena, sino lo que constituye el "único contenido de lo que aparece": ${ }^{55}$ en la mutación absoluta, el cambio constante y continuo, en el juego de las fuerzas, aparece lo que no cambia en todo cambio, en toda aparición y desaparición: la diferencia, no como una diferencia particular, lo cual llevaría de nuevo a la contraposición de fuerzas, sino la diferencia universal que resulta de la reducción -en el sentido más estrictamente fenomenológico"de las múltiples contraposiciones". ${ }^{56}$ Lo interior queda así colmado, constituido y re-llenado -el prefijo hace referencia al movimiento de la reflexión permanente de la realidad como fuerza- por la diferencia. Es el juego de la fuerza misma la que instituye la diferencia como diferencia de toda identidad y, por tanto, como diferencia universal. Esta diferencia universal se puede considerar, por ello, como "la ley de la fuerza"57 (Gesetz der Kraft). La fuerza, que es continua y permanente contraposición que se supera entre ser medio universal y unidad negativa, sigue así en su aparecer una ley: la de la diferencia. No es el fenómeno puro caos en su aparecer, sino que, como fenómeno, está sujeto a una ley, pero una ley que no lo identifica con un algo en concreto, sino con la ley que lo diferencia de todo lo que sensiblemente aparece. La ley de la diferencia muestra así en la negación de toda identificación una identidad, a saber: la identidad de la diferencia como diferencia. Es esta identidad de la que todavía, como sabemos, no es consciente plenamente la conciencia, porque todavía la conciencia no es razón, la que se nos aparece como "la imagen constante del fenómeno inestable". ${ }^{58}$ En un mundo lleno de fenómenos que aparecen y desaparecen, las leyes científicas de la naturaleza ofrecen para el entendimiento la posibilidad de que los fenómenos de la naturaleza aparezcan como estables y permanentes. Esto corresponde a la imagen de un "tranquilo reino de leyes" 59 que ahora, en el cenit de la ciencia moderna, ha ocupado el lugar que otrora ocuparon las simples ideas del mundo de las ideas. Será ahora, en este mundo

\footnotetext{
55 Ibíd.

56 Ibíd., p. 92.

57 Ibíd.

58 Ibíd.

59 Ibíd.
} 
conformado por las leyes de la naturaleza, en el que la conciencia tenga de otro modo que hacer experiencia de ese objeto del entendimiento que es el universal incondicionado.

Ahora bien, la ley, que aparece de modo absoluto y universal en la lógica, es de hecho una ley en la naturaleza que, a su vez, está en relación con una pluralidad de leyes. El objeto del entendimiento es ahora lo universal mismo en las leyes que rigen la naturaleza dándole orden. Sigue tratándose de un universal incondicionado - las leyes de la naturaleza no tienen contenido sensible alguno- pero, sin embargo, este universal ya no es tampoco el de la ley lógica de la pura diferencia que hace posible cualquier aparecer. Ahora se trata, más bien, del modo como se nos aparece la naturaleza como fenómeno en sí mismo a través de leyes científicas. Pero, al hablar de la naturaleza, ¿podemos considerarla un fenómeno o, por el contrario, hemos de asumir la radical diferencia de los fenómenos de la naturaleza? Esta cuestión se traduce, como puede verse, en la siguiente: ¿Pueden o no reducirse las diferentes leyes de la naturaleza descubiertas por el entendimiento a una única ley o, por el contrario, hemos de asumir que esa reducción no es posible? Si no lo es, ¿puede significar esto que esa pluralidad sea ya como tal lo que constituye el ámbito subyacente de los fenómenos naturales, o bien que nuestro entendimiento aún no ha sido capaz de penetrar suficientemente en las más duras capas de la naturaleza para ver cómo se origina todo fenómeno?

Pues bien, para Hegel, este conjunto de leyes que él piensa como un reino tranquilo es sólo la primera verdad del objeto del entendimiento. Ese reino de leyes no lleva a su cumplimiento el fenómeno o no agota totalmente la manifestación. La ley de la fuerza no puede llenar el fenómeno mismo. "La ley - dice Hegel- se halla presente en él, pero no es toda su presencia (ihre ganze Gegenwart)". ${ }^{60}$ La ley está en el fenómeno constituyéndolo qua fenómeno, pero la ley per se no lo llena de presencia. Bajo la lógica hegeliana, esa primera verdad, por ser todavía demasiado abstracta, está necesitada, a su vez, de otra que, de alguna manera, la niegue, supere y retenga. Que se da esta otra, viene recogido en la siguiente afirmación de Hegel: "Bajo circunstancias sin cesar distintas, tiene una realidad siempre otra". ${ }^{61}$ El fenómeno en circunstancias distin-

\footnotetext{
${ }^{60}$ Fenomenología del espíritu, cit., p. 92.

${ }^{61}$ Ibíd. 
tas (anderen Umständen) tiene siempre otra realidad efectiva (Wirklichkeit). La efectividad del fenómeno, de su aparición, depende también de las circunstancias en la que aparece. Esta apelación a las circunstancias es lo que nos hace pensar que estamos en el reino o mundo de la naturaleza: la naturaleza, en su manifestación, está sujeta a las circunstancias. Por tanto, aquella ley del fenómeno como ley de la fuerza no puede por sí sola dar cuenta del fenómeno como fenómeno y de todo el contenido de su presencia. La realidad efectiva del fenómeno en su aparición no obedece sólo a la determinación de la ley. Y esta menesterosidad de la ley, este no poder describir cabalmente cómo el fenómeno se manifiesta, ha de tener, según Hegel, un reflejo en la ley misma. Efectivamente, esto que le falta a la ley para dar razón del fenómeno, viene expresado, en un nuevo momento para la conciencia, como el paso para el entendimiento de la consideración de la ley a una ley, es decir como una ley que no puede determinarse como la ley. Positivamente, si hay una ley, hay multiplicidad de leyes: si se toma una también puede tomarse otra. Pero, esto mismo, el introducir de nuevo la multiplicidad como objeto del entendimiento, es algo que contradice el principio del entendimiento, que como tal tiene que ver simplemente el objeto, esto es, verlo como lo universal incondicionado. A diferencia de la sensibilidad, como ya sabemos, el entendimiento no puede ver la multiplicidad sino a través de la unidad subyacente de esa multiplicad. En el terreno de las leyes de la naturaleza, la búsqueda de esta unidad lleva al entendimiento a querer reducir las leyes a una sola ley. La conciencia parece encontrarla en la ley de la gravitación de Newton: "El entendimiento supone haber descubierto aquí una ley universal que expresa la realidad universal como tal, pero sólo ha descubierto, de hecho, el concepto de la ley misma, algo así como si declara que toda realidad es en ella misma conforme a la ley". ${ }^{62}$

La importancia de esta ley concreta es que en ella la conciencia ha hecho experiencia del concepto mismo de ley en el ámbito de la naturaleza y ha hecho concepto en tanto que esta ley expresa en sí misma que la naturaleza es conforme a la ley o "que toda realidad efectiva es legaliforme en ella misma". ${ }^{63}$ La realidad es conforme a ley, está sujeta a una ley que pertenece y obra dentro de la naturaleza misma y que ha sido expuesta desde el trato mismo de la con-

62 Ibíd., p. 93.

${ }^{63}$ Fenomenología del espíritu, traducción de A. Gómez Ramos, op. cit., p. 223. 
ciencia con los fenómenos de la naturaleza. Nunca antes de Newton se tuvo, como señala Hegel en los ejemplos, una ley que explicara, a partir de la fuerza, el movimiento de manera universal. Por ello, la importancia de esta ley está en que con ella se destruye toda "representación carente de pensamiento para la que todo se presenta bajo la figura contingente y según la cual la determinabilidad tiene la forma de la independencia sensible". ${ }^{64}$ Efectivamente, la mecánica de Newton rompe con la formulación de las leyes que se apoyaban en lo sensible o empírico y, sin embargo, ello no le lleva a pro-poner un mundo metafísico. Esta ruptura se produce cuando las leyes concretas, que explicaban fragmentariamente la naturaleza, fueron negadas en su determinidad por una única ley universal que no pertenece, como decimos, al terreno del noúmeno, de lo suprasensible, sino al terreno del fenómeno sin dejar de ser por ello necesaria. Efectivamente, la conciencia tiene aquí la experiencia de que las diferencias en la ley "regresan ellas mismas a lo interior en cuanto unidad simple". ${ }^{65}$ En este retorno a la unidad simple se cifra la necesidad de la ley. En lo que, sin duda, es la parte más extraña de este texto, aquí Hegel intentará buscar en la ley los mismos momentos de la diferencia que aquellos que ya aparecieron en la fuerza. Hegel toma el ejemplo de la electricidad como una fuerza simple pero que aparece al mismo tiempo en dos momentos diferentes: el polo positivo y el polo negativo. La diferencia de la ley aparece ahora de forma inmediata en estos dos lados: de uno, en la fuerza como la forma simple de lo que ya ha vuelto dentro sí y lo que, por tanto, permanece en la identidad; pero, de otro, como los momentos en sí diferentes cuando sale fuera de sí. ¿Hay una necesidad entre estos momentos expresados por la ley y la simplicidad de fuerza? ¿Hay en la ley que expresa esta diferencia un vínculo necesario entre la identidad y la diferencia? ¿Hay necesidad entre la simplicidad de la fuerza como tal y los polos en que se exterioriza? Parece que tal necesidad se da en tanto que la fuerza, de exteriorizarse, de desdoblarse, sólo puede hacerlo así y no de otra manera.

Pero, ahora bien, esta argumentación de Hegel está encaminada a mostrar que aquí no hay una verdadera necesidad, y no la hay porque esa relación que se expresa en la ley es sólo un factum físico: "la fuerza debe desdoblarse así

${ }^{64}$ Ibíd., p. 93.

${ }^{65}$ Fenomenología del espíritu, trad. de A. Gómez Ramos, op. cit., p. 223.

| Investigaciones Fenomenológicas, n. 13, 2016. 
sencillamente porque debe". ${ }^{66}$ No hay necesidad lógica entre la simple fuerza y los momentos en que se despliega. Para la electricidad como fuerza simple es indiferente que aparezca como positiva y negativa. Por ello, la verdadera necesidad, el verdadero vínculo necesario sólo puede venir dado como exigencia del concepto, pero de un concepto que ya lleva dentro de sí, como su esencia, el ser de la cosa. Y esto no se da en el concepto según la ley de la naturaleza. El entendimiento conoce esta diferencia, tiene el concepto de esta diferencia, pero, todavía, como sabemos nosotros, no tiene el concepto de este concepto, y no conoce, por tanto, la "diferencia de la cosa misma". 67 Por ello, en la explicación (Erklären), como clarificación, se expone sólo "la descripción de los momentos que forman el círculo de la necesidad". ${ }^{68}$ De esta manera, se hace coincidir la cosa, la fuerza, con el contenido mismo de la ley. Así puede decir Hegel que las explicaciones no dejan de tener, vistas desde la necesidad del concepto, un carácter tautológico: "En la cosa misma no nace con este movimiento nada nuevo". ${ }^{69}$ Las diferencias que se ponen en este movimiento no son diferencias de la cosa en sí misma, no son realmente efectivas y, en tanto que no lo son, pueden ser consideradas como diferencias que en sí mismas están llamadas a ser canceladas y superadas.

Verdaderamente, en el ámbito de las leyes de la naturaleza no se da una verdadera necesidad. Esto queda ejemplarmente expuesto en las críticas que Hegel lleva a cabo a propósito de la ley de la gravedad de Newton. Como ha señalado Ihmig, la crítica de Hegel a Newton - no a sus seguidores británicos como Martin y Maclaurin o los fisicistas franceses como D'Alembert, Laplace o Lagrange- no está totalmente injustificada. Efectivamente, Hegel habría señalado el uso no siempre consistente de los conceptos en el propio Newton, como, por ejemplo, el de la fuerza de la inercia que concibe como una fuerza innata similar al concepto de impetus postulado en la Edad Media, ${ }^{70}$ o como la aleatoria identificación que hace Newton entre la fuerza centrípeta o fuerza atractiva y la gravitación. ${ }^{71}$ Detengámonos un momento aquí.

\footnotetext{
${ }^{66}$ Ibíd., p. 94.

${ }^{67}$ Ibíd., p. 96.

68 Ibíd.

${ }^{69}$ Ibíd.

${ }^{70}$ Cf. K-N. Ihmig, "Hegel's rejection of the concept of force" en M. J. Petry (ed.), Hegel and Newtonanism. Dordrecht: Springer Netherlands, p.405.

${ }^{71}$ Cf. Ibíd., p. 404.
} 
El problema surge en torno a la reconstrucción del movimiento de los planetas sobre la base del paralelogramo de fuerzas en la primera proposición de la sección segunda de los Principia donde Newton establece la relación entre la segunda ley de Kepler y la fuerza centrípeta. Hegel rechaza el concepto de fuerza que aquí Newton pone en juego en tanto que este no distinguiría "entre la construcción matemático-geométrica de la prueba y su aspecto físicodinámico". ${ }^{72}$ Para Hegel, habría que distinguir ontológicamente la reconstrucción matemática del movimiento de la interpretación física del mismo para no acabar deduciendo, en este caso, las fuerzas físicas efectivas de las líneas geométricas en la construcción del paralelogramo de las fuerzas. Como señala Ihmig, Newton no trazó esta diferencia fundamental "entre la medida empírica de la constante de la caída sobre la superficie de la tierra y la determinación cuantitativa de la gravitación universal...". ${ }^{73} \mathrm{Y}$ es que para Hegel la ley de la gravitación ha de ser diferente a una constatación empírica, es decir, ha de ser considerada universalmente, esto es, filosófica o especulativamente, sin que quede rebajada a ser la expresión de una medida. En la Enciclopedia, en el parágrafo 269, afirmará esta tesis con las siguientes palabras: "La gravitación es el concepto verdadero y determinado de la corporeidad material que se ha realizado hasta [alcanzar] la idea". ${ }^{74}$ En la Ciencia de la lógica, llegará a decir Hegel que las pruebas newtonianas constituyen un armazón vacío porque las matemáticas en general no pueden probar las determinaciones de la magnitud de la física en tanto que estas determinaciones tienen como fundamento una naturaleza cualitativa - no cuantitativa - que sólo puede venir dada mediante el concepto, es decir, mediante el saber de la filosofía. ${ }^{75}$ Por ello, para Hegel, sólo si la ley de la gravitación como una ley de la fuerza se da según el concepto, puede la conciencia hacer experiencia en ella de lo universal como aquello absoluto que mantiene en relación todo lo que hay desde sí mismo, desde su interior como la fuerza que lo constituye. En este sentido lo universal tiene que darse con la necesidad propia del concepto.

\section{CONCLUSIÓN}

\footnotetext{
${ }^{72}$ Ibíd., p. 402.

73 Ibíd., p. 408.

${ }^{74}$ G. W. F. Hegel, Enciclopedia de las ciencias filosóficas, Madrid: Alianza Editorial, 1999, p. 331.

75 G. W. F. Hegel, Ciencia de la lógica, Madrid: Abada Editores/UAM Ediciones, 2011, p. 369.
} 
Hemos visto que, con la dialéctica de las leyes en el terreno del entendimiento y con la dialéctica de la ley en el interior de ella misma, el movimiento, que antes parecía pertenecer solamente al mundo sensible, ha llegado al mundo inteligible o al interior del fenómeno. Vemos, por tanto, ahora, en el objeto del entendimiento, en sus diferentes momentos, el mismo problema con el que antes nos habíamos encontrado en el terreno del mundo sensible: ese reino tranquilo e inmarcesible de las leyes ha sido derrumbado por este movimiento que amenazaba ya desde su cuna la supuesta soberanía absoluta en la que la ley parecía descansar. Por tanto, se hace necesario otro concepto de ley desarrollado por el saber de la filosofía que pueda dar cuenta de lo que efectivamente hace posible todo aparecer. Las leyes de la naturaleza son un momento necesario, pero un momento al fin y al cabo, que tiene que ser visto, negado, elevado e integrado por el saber de la filosofía como un saber lógico/metafísico. Pero, para ello, volviendo al texto que abría este capítulo, donde nosotros reiterábamos la experiencia anterior y vislumbrábamos la venidera, vemos que hay que pasar de la conciencia en la figura del entendimiento a la figura de la autoconciencia. "La conciencia - dice Hegel- no es todavía para sí misma el concepto, por lo cual no se reconoce en aquel objeto reflejado". ${ }^{76}$ El entendimiento lleva a cabo su tarea de penetrar en su objeto como aquello que constituye la relacionabilidad en la que están todas las cosas, pero todavía el entendimiento no es conciencia de sí mismo, o, mejor expresado, todavía no es conciencia que tiene conciencia de sí como de un cierto saber. Ahora bien, el paso a la autoconciencia requiere todavía de un momento más que aquí ya no puede ser desarrollado: aquel en el que la conciencia experiencia que aquel mundo suprasensible, que está animado por el cambio en el interior de sí mismo en virtud de la diferencia pero que no obedece todavía a un principio lógico que da razón de lo efectivamente real en su aparecer, se con-vierte en lo otro de sí, en lo contrario de sí, en la contraimagen de ese mundo suprasensible donde la ley del aparecer torna o cambia lo que aparece en su contrario, y de tal modo que cada imagen del mundo suprasensible tiene su igual imagen contrapuesta en el mundo invertido (werkehrte Welt) y no puede, por ello, ser considerado como una simple manifestación del mundo suprasensible, sino como otro mundo de

\footnotetext{
${ }^{76}$ Fenomenología del espíritu, cit., p. 82.
} 
igual rango ontológico que este y que viene, por tanto, a negar la diferencia misma entre un mundo verdadero y otro falso, pero que reafirma, en el interior del mundo suprasensible mismo, una diferencia absoluta que es irreductible para la conciencia y que es experienciada por ella como la infinitud de la vida simple de la naturaleza. ${ }^{77}$ Es de este hundimiento de la conciencia en el entendimiento de esta infinitud de donde surgirá la diferencia de la propia conciencia como conciencia que apunta intencionalmente a lo otro de sí de la naturaleza: a un concepto de vida, ya no perteneciente al mundo natural sino al espíritu, el cual tiene su origen, como ya hemos señalado, en el desarrollo de la figura de la autoconciencia. Pero para ello, como dice en una bella imagen Hegel, ${ }^{78}$ hay cerrar el telón que se alza sobre lo interior, pues en este interior ya no hay nada que ver a menos que nosotros mismos entremos en él. Ciertamente, en la experiencia del mundo invertido, el fenómeno, como concepto de la totalidad de lo que aparece, se consuma ${ }^{79}$ y con él el contenido de lo que se nos muestra en el fenómeno se niega, se supera y se abre a las manifestaciones del espíritu que apuntan a la necesidad de un concepto lógico de fenómeno que dé razón de la totalidad de lo que aparece, tanto en la ya siempre naciente pero reglada naturaleza como en el ya siempre naciente y libérrimo espíritu.

\footnotetext{
77 Para la cuestión del mundo invertido y el paso a la infinitud de la vida véase: H. G. Gadamer, op. cit., pp. 49-74; M. Heidegger, op. cit., pp. 214-218.

${ }_{78}$ Cf. Fenomenología del espíritu, cit., p. 104.

${ }^{79}$ Cf. Ibíd.

Investigaciones Fenomenológicas, n. 13, 2016.
} 